\title{
SPECIES COMPOSITION AND ABUNDANCE OF ROTIFERS IN DIFFERENT ENVIRONMENTS OF THE FLOODPLAIN OF THE UPPER PARANÁ RIVER, BRAZIL
}

\author{
Aparecida Paula Perez Garcia ${ }^{1}$ \\ Fábio Amodêo Lansac-Tôha ${ }^{1}$ \\ Cláudia Costa Bonecker ${ }^{1}$
}

\begin{abstract}
The purpose of this study was to describe the composition and abundance of rotifers in different environments: one lotic (the Ivinheima River) and another lentic (the Patos Lake) located in the floodplain of the Upper Paraná River. The influence of limnological variables on the structure and dynamics of rotifers was also examined. Samples were taken monthly from March 1992 through February 1993, in the open water region of the lake and river and in the channel between them and, in the littoral region of the lake and river. Of the 96 species identified, the best represented families were: Brachionidae ( 20 species), Lecanidae (18 species), and Trichocercidae (15 species). 55 species were widely distributed, 13 were pantropical, 16 neotropical ( 8 endemic to South America). Trichocerca gracilis was a new record for this floodplain. Dipleuchlanis propatula propatula, Lecane curvicornis, L. leontina, $L$. bulla, Plationus patulus patulus, Platyias quadricornis quadricornis, Testudinella patina, and T. mucronata hauriensis were consistently present. More species were recorded during the high water period, probably because of the interconnection among the different environments of the floodplain that allows fauna exchange and an increase in available habitats. Rotifer densities were higher mainly in the lake, during the high water. Using principal components analysis, was identified four variables that influence the seasonal variation in the rotifer densities: water level, dissolved oxygen, chlorophyll-a concentration, and water temperature.

KEY WORDS. Rotifera, Paraná River, floodplain, neotropics
\end{abstract}

On a floodplain, the seasonal variation in the hydrological conditions, characterized by the occurrence of high water and low water periods, is a fundamental ecological factor. Water level variations cause changes in the abiotic and biotic features of the whole system and regulate the exchange of matter and energy between the main river and the adjacent environments (JUNK et al. 1989).

The Paraná River has areas of floodplain in some of its stretches; in this environment and others, the zooplankton community varies in its composition and abundance in relation to changes in physical, chemical, and biological characteristics (VÁSQuez 1984a,b; RoBERTSON \& HARDY 1984; PAGGI \& JOSÉ DE PAGGI 1990; LANSAC-TÔHA et al. 1997). Among the zooplankton, the rotifers are notably most the abundant and diverse. This community richness, allied to their high turnover, indicates the ecological importance of rotifers in energy flow and nutrient cycling (ALLAN 1976).

1) Curso de Pós-graduação em Ambientes Aquáticos Continentais, Departamento de Biologia, Nupélia, Universidade Estadual de Maringá. Avenida Colombo 5790, 87020-900 Maringá, Paraná, Brasil. 
The aim of this paper is to describe the composition and abundance of rotifers in a lotic environment (Ivinheima River) and a lentic environment (Patos Lake), located in the floodplain of the Upper Paraná River, Mato Grosso do Sul State, as well as to identify the influence of physical, chemical, and biological variables on the rotifer community structure and dynamics.

\section{MATERIALS AND METHODS}

\section{Study area}

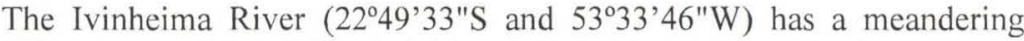
pattern and turbulent waters. Its margins are covered by grasses (Polygonon sp.) and, in part, occupied by aquatic macrophytes (Eichhornia azurea Kunth). The Ivinheima communicates with the Paraná River by a channel (Fig. 1).

The Patos lake $\left(22^{\circ} 43^{\prime} 12^{\prime \prime} \mathrm{S}\right.$ and $\left.53^{\circ} 17^{\prime} 37^{\prime \prime} \mathrm{W}\right)$ is located on the right bank of the Paraná River and on the left bank of the Ivinheima River, communicating with the latter throughout the year by a channel. This channel contains large beds of aquatic macrophytes along its length and its bank is dominated by grasses. The flow direction and water speed in the channel vary according to the flood stage of the river. The irregular shore at the lake is covered by grasses and small beds of aquatic macrophytes, predominantly E. azurea (Fig. 1).

\section{Sampling stations}

For this study, was established five sampling stations (Fig. 1): two in the lake (Stations 1 -open water region and 2 - littoral region), one in the channel between the river and the lake (Station 3 - open water), and two in the river (Stations 4 littoral region and 5 - open water region).

\section{Physical, chemical, and biological variables}

We collected monthly samples for physical, chemical, and biological variables, always during the morning, from March 1992 to February 1993, at all stations. At stations 1 and 3 were collected the samples at three depths of the water column (surface, middle, and bottom), at station 5, at two depths (surface and bottom), and at stations 2 and 4 , only on the surface.

Water level data for the Paraná River were supplied by DNAEE (National Department of Water and Energy). Analyses of the abiotic and biotic variables: water temperature $\left({ }^{\circ} \mathrm{C}\right)$, water column transparency $(\mathrm{m})$, dissolved oxygen $(\mathrm{mg} / \mathrm{l})$, $\mathrm{pH}$, electrical conductivity $(\mu \mathrm{S} / \mathrm{cm})$, and chlorophyll-a concentration $(\mathrm{mg} / \mathrm{l})$ were conducted according to the methodology described by THOMAZ et al. (1992).

\section{Rotifers}

We obtained the rotifer samples with a motorized pump filtering 1000 liters of water for each sample through a $70 \mu \mathrm{m}$ mesh plankton net.

Rotifers (ind $/ \mathrm{m}^{3}$ ) were quantified by counting sub-samples in a SedgwickRafter cell; at least 200 individuals of each sample were counted. Identification was based on Koste (1978), Koste \& RoberTSON (1983) and SEgers (1995). 


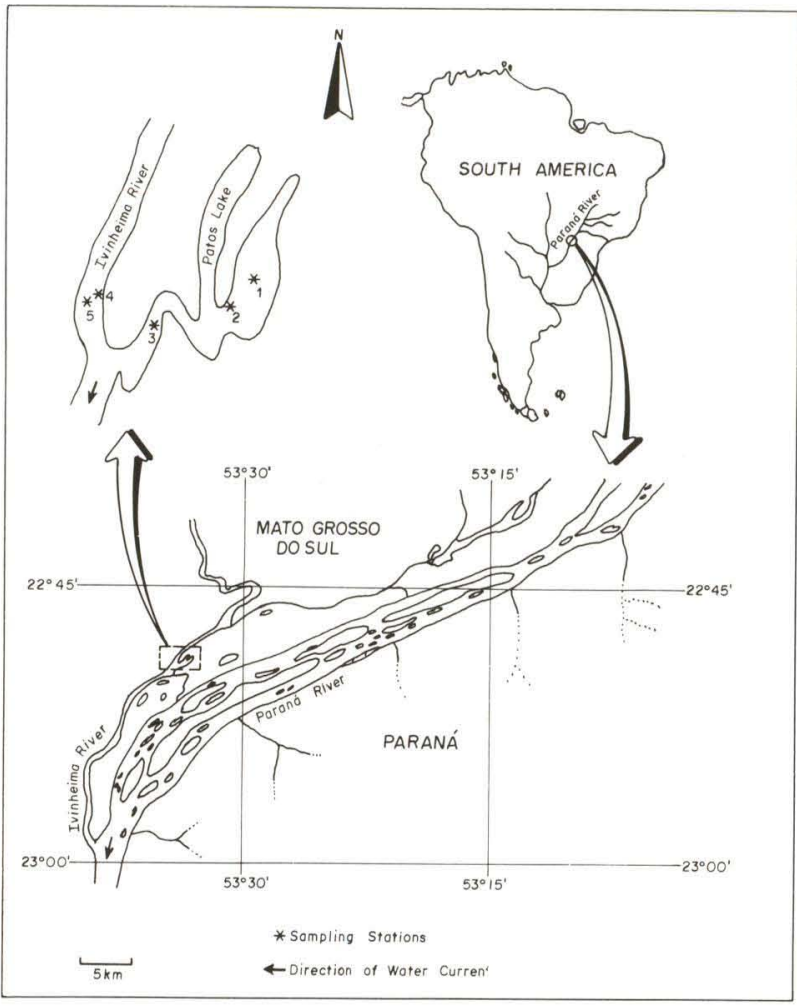

Fig. 1. Sampling stations.

\section{Constancy Index and Geographical Distribution}

A Constancy Index (c) for each species recorded at the five sampling stations, was determined by the expression $\mathrm{c}=(\mathrm{n} \times 100) / \mathrm{N}$, where " $\mathrm{n}$ " is the number of samples containing the species and "N" is the total number of samples collected (DAJOZ 1973). According to the results of this index, the species were considered constant when they occurred in more than $50 \%$ of the samples; accessory, when recorded in $25 \%-50 \%$ of the samples, and accidental when present in less than $25 \%$ of the samples.

From the data on geographic distribution for each species (KOSTE 1978; JosÉ DE PAGGI 1990; BONECKER et al. 1994), the species were classified as having a widespread distribution, as pantropical, neotropical, or neotropical and endemic to South America.

\section{Principal components analysis (PCA)}

To evaluate the influence of the temporal and spatial variation of the environmental factors on the total densities of the rotifers and the principal species, were conducted a multivariate analysis of the data. PCA examines the interdependence among the variables and, from the data, discovers models that allow us to formulate hypothesis as a function of the estimated variables (PLA 1986). 
From this analysis was derived a data matrix with all the limnological variables (Tabs I, II). The data, except for $\mathrm{pH}$, were $\log$ transformed $[\log (\mathrm{x}+1)]$, and standardized since were dealing with variables with distinct units of measurement.

PCA was performed using Statistica software (STATSOFT 1991). To interpret the analysis was considered the axis that explained in total $50 \%$ or more of the variability of the data. Only those variables with structure coefficients $=0.5$ were considered.

Table I. Water level of the Paraná River. Average values (x), standard deviation (s), maximum ( $\max )$ and minimum (mim) environmental variables obtained at five sampling stations in March 1992 - February 1993. For stations 2 and 5 only surface data are presented. These data were used in the PCA.

\begin{tabular}{|c|c|c|c|c|c|c|c|c|c|c|c|c|}
\hline Variables per Station & Mar & Apr & May & Jun & Jul & Aug & Sep & Oct & Nov & Dec & Jan & Feb \\
\hline Water level $(\mathrm{m})$ & 4.20 & 4.60 & 4.80 & 3.44 & 2.80 & 2.91 & 2.88 & 3.23 & 4.42 & 4.77 & 4.13 & 5.36 \\
\hline \multicolumn{13}{|l|}{ Station 1} \\
\hline Depth $(m)$ & 4.20 & 4.60 & 6.60 & 5.80 & 4.20 & 3.60 & 3.80 & 4.40 & 4.80 & 5.60 & 4.00 & 4.60 \\
\hline Water transparency $(\mathrm{m})$ & 1.20 & 0.80 & 0.50 & 1.40 & 1.70 & 0.90 & 0.60 & 0.60 & 1.10 & 1.80 & 1.40 & 1.00 \\
\hline Water temperature $\left({ }^{\circ} \mathrm{C}\right) \bar{x}$ & 26.43 & 25.56 & 23.96 & 23.36 & 18.13 & 18.30 & 20.80 & 23.36 & 26.53 & 29.10 & 26.83 & 27.93 \\
\hline s & 0.58 & 0.05 & 0.05 & 0.15 & 0.30 & 0.20 & 0.26 & 0.11 & 1.45 & 0.36 & 0.25 & 0.20 \\
\hline Max & 27.10 & 25.60 & 24.00 & 23.50 & 18.40 & 18.50 & 21.00 & 23.50 & 28.00 & 29.40 & 27.10 & 28.10 \\
\hline Min & 26.00 & 25.50 & 23.90 & 23.20 & 17.80 & 18.10 & 20.50 & 23.30 & 25.10 & 28.70 & 26.60 & 27.70 \\
\hline Dissolved oxygen (mg/l) & 55.50 & 22.43 & 27.66 & 16.36 & 37.13 & 94.90 & 93.83 & 16.60 & 67.36 & 18.43 & 46.40 & 25.90 \\
\hline s & 0.58 & 0.05 & 0.05 & 0.15 & 0.30 & 0.20 & 0.26 & 0.11 & 1.45 & 0.36 & 0.25 & 0.20 \\
\hline $\operatorname{Max}$ & 67.60 & 24.00 & 29.50 & 17.40 & 41.70 & 97.00 & 96.40 & 18.20 & 97.80 & 23.10 & 50.30 & 27.90 \\
\hline Min & 32.60 & 20.70 & 25.50 & 14.70 & 30.20 & 92.40 & 90.70 & 13.90 & 32.00 & 14.40 & 43.50 & 22.50 \\
\hline Electrical conductiv & 49.33 & 40.66 & 35.00 & 45.66 & 39.66 & 31.66 & 33.00 & 41.33 & 38.33 & 36.00 & 36.00 & 37.00 \\
\hline s & 3.21 & 0.57 & 0.00 & 1.52 & 0.57 & 1.15 & 0.00 & 0.57 & 1.52 & 0.00 & 0.00 & 0.00 \\
\hline Max & 53.00 & 41.00 & 35.00 & 47.00 & 40.00 & 33.00 & 33.00 & 42.00 & 40.00 & 36.00 & 36.00 & 37.00 \\
\hline Min_ & 47.00 & 40.00 & 35.00 & 44.00 & 39.00 & 31.00 & 33.00 & 41.00 & 37.00 & 36.00 & 36.00 & 37.00 \\
\hline $\mathrm{pH} \bar{x}$ & 6.60 & 6.10 & 6.26 & 6.13 & 6.23 & 6.36 & 7.50 & 6.33 & 6.76 & 6.43 & 6.53 & 6.40 \\
\hline s & 0.14 & 0.00 & 0.11 & 0.05 & 0.05 & 0.15 & 0.17 & 0.05 & 0.11 & 0.05 & 0,05 & 0.00 \\
\hline Max & 6.70 & 6.10 & 6.40 & 6.20 & 6.30 & 6.50 & 7.70 & 6.40 & 6.90 & 6.50 & 6.60 & 6.40 \\
\hline Min & 6.50 & 6.10 & 6.20 & 6.10 & 6.20 & 6.20 & 7.40 & 6.30 & 6.70 & 6.40 & 6.5 & 6.40 \\
\hline Chlorophyll -a ( $\mu \mathrm{g} / \mathrm{l})$ & 2.73 & 3.21 & 1.91 & 1.09 & 1.21 & 5.27 & 28.91 & 4.12 & 6.96 & 2.65 & 8.5 & 5.46 \\
\hline s & 1.44 & 2.78 & 1.93 & 0.00 & 0.60 & 0.31 & 5.50 & 1.16 & 0.49 & 0.72 & 0.34 & 1.82 \\
\hline Max & 4.37 & 6.37 & 3.28 & 1.09 & 1.82 & 5.46 & 33.70 & 5.46 & 7.51 & 3.12 & 8.87 & 7.28 \\
\hline $\operatorname{Min}$ & 1.64 & 1.09 & 0.55 & 1.09 & 0.61 & 4.91 & 22.93 & 3.28 & 6.55 & 1.82 & 8.19 & 3.64 \\
\hline \multicolumn{13}{|l|}{ Station 2} \\
\hline Depth $(m)$ & & 2.20 & 3.20 & & 1.20 & 1.00 & 1.40 & 1.80 & 2.00 & 2.80 & 1.40 & 2.00 \\
\hline Water transparency $(\mathrm{m})$ & 1.30 & 0.80 & 0.50 & 1.70 & 1.10 & 0.70 & 0.60 & 0.70 & 1.10 & 1.30 & 1.10 & 1.00 \\
\hline Water temperature & 28.10 & 25.40 & 24.10 & 23,40 & 18.70 & 18.60 & 20.60 & 23.70 & 27.80 & 29.30 & 26.70 & 28.30 \\
\hline Dissolved oxygen & 66.50 & 31.40 & 28.50 & 20.00 & 44.201 & 101.80 & 120.80 & 36.60 & 76.80 & 18.10 & 23.70 & 27.70 \\
\hline Electrical conductivity $(\mu \mathrm{S} / \mathrm{cm})$ & 49.00 & 38.00 & 35.00 & 48.00 & 40.00 & 32.00 & 33.00 & 41.00 & 38.00 & 36.00 & 37.00 & 37.00 \\
\hline $\mathrm{pH} \bar{x}$ & 6.70 & 6.00 & 6.40 & 6.20 & 6.40 & 6.50 & 7.50 & 6.40 & 6.90 & 6.40 & 6.40 & 6.40 \\
\hline Chlorophyll -a ( $\mu g / l)$ & 0.00 & 3.28 & 0.55 & 0.55 & 2.43 & 5.46 & 32.22 & 6.55 & 6.67 & 2.73 & 7.89 & 3.64 \\
\hline \multicolumn{13}{|l|}{ Station 3} \\
\hline Depth $(m)$ & 4.40 & 5.00 & 7.00 & 6.40 & 4.40 & 4.00 & 4.40 & 4.80 & 5.20 & 5.00 & 4.40 & 5.00 \\
\hline Water transparency $(\mathrm{m})$ & 1.10 & 0.90 & 0.50 & 1.40 & 1.10 & 1.00 & 0.60 & 0.70 & 1.00 & 1.40 & 1.10 & 1.00 \\
\hline Water temperature $\left({ }^{\circ} \mathrm{C}\right) \bar{x}$ & 26.80 & 25.73 & 23.96 & 23.30 & 19.13 & 17.86 & 20.46 & 23.66 & 26.83 & 29.26 & 27.83 & 27.96 \\
\hline s & 0.87 & 0.11 & 0.05 & 0.17 & 0.11 & 0.35 & 0.49 & 0.23 & 0.70 & 0.23 & 0.35 & 0.05 \\
\hline Max & 27.80 & 25.80 & 24.00 & 23.40 & 19.20 & 18.20 & 20.80 & 23.80 & 27.60 & 29.40 & 28.20 & 28.00 \\
\hline Min & 26.20 & 25.60 & 23.90 & 23.10 & 19.00 & 17.50 & 19.90 & 23.40 & 26.20 & 29.00 & 27.50 & 27.90 \\
\hline Dissolved oxygen $(\mathrm{mg} / \mathrm{l}) \bar{x}$ & 72.73 & 33.16 & 36.76 & 22.33 & 55.601 & 100.301 & 108.80 & 26.40 & 72.30 & 18.90 & 59.30 & 14.83 \\
\hline s & 21.23 & 0.20 & 1.17 & 1.00 & 2.66 & 1.10 & 4.42 & 0.34 & 10.71 & 0.17 & 1.69 & 0.75 \\
\hline Max & 95.20 & 33.40 & 38.10 & 23.10 & 58.601 & 101.401 & 112.70 & 26.60 & 81.20 & 19.00 & 60.50 & 15.70 \\
\hline Min & 53.00 & 33.00 & 35.90 & 21.20 & 53.50 & 99.201 & 104.00 & 26.00 & 60.40 & 18.70 & 58.10 & 14.40 \\
\hline
\end{tabular}

Cont. 
Table I. Continued.

\begin{tabular}{|c|c|c|c|c|c|c|c|c|c|c|c|c|}
\hline Variables per Station & Mar & Apr & May & Jun & Jul & Aug & Sep & Oct & Nov & Dec & Jan & Feb \\
\hline Electrical conductivity $(\mu \mathrm{S} / \mathrm{cm}) \bar{x}$ & 47.60 & 40.60 & 35.30 & 47.00 & 42.30 & 41.00 & 40.00 & 44.30 & 45.30 & 36.30 & 36.60 & 40.00 \\
\hline s & 1.52 & 0.57 & 0.57 & 0.00 & 0.57 & 2.64 & 0.00 & 0.57 & 0.57 & 0.57 & 0,57 & 1.00 \\
\hline Max & 49.00 & 41.00 & 36.00 & 47.00 & 43.00 & 43.00 & 40.00 & 45.00 & 46.00 & 37.00 & 37.00 & 41.00 \\
\hline Min & 46.00 & 40.00 & 35.00 & 47.00 & 42.00 & 38.00 & 40.00 & 44.00 & 45.00 & 36.00 & 36.00 & 39.00 \\
\hline $\mathrm{pH} \bar{x}$ & 6.60 & 6.16 & 6.16 & 6.33 & 6.53 & 6.83 & 7.16 & 6.50 & 6.80 & 6.53 & 6.63 & 6.40 \\
\hline s & 0.10 & 0.05 & 0.05 & 0.11 & 0.05 & 0.05 & 0.05 & 0.10 & 0.00 & 0.15 & 0.05 & 0.00 \\
\hline Max & 6.70 & 6.20 & 6.20 & 6.40 & 6.60 & 6.90 & 7.20 & 6.60 & 6.80 & 6.70 & 6.70 & 6.40 \\
\hline Min & 6.50 & 6.10 & 6.10 & 6.20 & 6.50 & 6.80 & 7.10 & 6.40 & 6.80 & 6.40 & 6.60 & 6.40 \\
\hline Chlorophyll -a $(\mu g / l) \bar{x}$ & 2.55 & 3.09 & 1.09 & 0.55 & 2.30 & 2.36 & 7.82 & 3.70 & 1.82 & 3.07 & 9.10 & 6.52 \\
\hline s & 2.57 & 0.62 & 0.00 & 0.00 & 0.84 & 0.62 & 1.37 & 0.91 & 1.37 & 1.27 & 0.52 & 1.83 \\
\hline Max & 5.46 & 3.82 & 1.09 & 0.55 & 3.28 & 2.73 & 9.28 & 4.55 & 3.28 & 4.37 & 9.71 & 8.19 \\
\hline Min & 0.55 & 2.73 & 1.09 & 0.55 & 1.82 & 1.64 & 6.55 & 2.73 & 0.55 & 1.82 & 8.74 & 4.55 \\
\hline \multicolumn{13}{|l|}{ Station 4} \\
\hline Depth $(m)$ & 3.00 & 2.00 & 4.60 & 4.20 & 3.00 & 1.60 & 1.80 & 2.60 & 5.50 & 3.20 & 2.60 & 3.00 \\
\hline Water transparency (m) & 0.50 & 0.80 & 0.50 & 1.70 & 0.80 & 0.80 & 0.60 & 0.40 & 0.90 & 1.20 & 0.60 & 0.60 \\
\hline Water temperature $\left({ }^{\circ} \mathrm{C}\right)$ & 26.70 & 25.30 & 24.30 & 23.30 & 18.20 & 18.90 & 20.40 & 23.80 & 27.30 & 29.20 & 28.00 & 28.50 \\
\hline Dissolved oxygen (mg/l) & 88.30 & 59.80 & 47.20 & 30.30 & 99.401 & 106.90 & 96.70 & 81.60 & 61.90 & 15.80 & 86.10 & 82.90 \\
\hline Electrical conductivity $(\mu \mathrm{S} / \mathrm{cm})$ & 41.00 & 43.00 & 37.00 & 47.00 & 50.00 & 45.00 & 41.00 & 42.00 & 43.00 & 37.00 & 46.00 & 44.00 \\
\hline $\mathrm{pH}$ & 6.60 & 6.10 & 6.60 & 6.20 & 6.80 & 6.70 & 7.10 & 6.80 & 6.70 & 6.40 & 7.00 & 6.90 \\
\hline Chlorophyll -a $(\mu \mathrm{g} / \mathrm{l})$ & 2.73 & 7.64 & 0.00 & 1.09 & 0.00 & 1.37 & 2.18 & 1.09 & 3.28 & 0.91 & 4.55 & 2.18 \\
\hline \multicolumn{13}{|l|}{ Station 5} \\
\hline Depth $(m)$ & 4.60 & 5.00 & 7.40 & 6.50 & 6.00 & 5.00 & 5.00 & 6.50 & 2.80 & 6.00 & 4.20 & 5.00 \\
\hline Water transparency $(\mathrm{m})$ & 0.40 & 0.90 & 0.50 & 1.10 & 0.80 & 0.70 & 0.60 & 0.40 & 0.80 & 0.90 & 0.50 & 0.50 \\
\hline Water temperature $\left({ }^{\circ} \mathrm{C}\right) \overline{\mathrm{x}}$ & 26.70 & 25.25 & 24.20 & 23.70 & 20.60 & 18.35 & 19.35 & 22.30 & 26.10 & 27.70 & 28.30 & 28.45 \\
\hline s & 0.14 & 0.07 & 0.00 & 0.07 & 3.39 & 0.07 & 1.62 & 2.82 & 2.54 & 0.07 & 0.14 & 0.07 \\
\hline Max & 26.80 & 25.30 & 24.20 & 24.20 & 23.00 & 18.40 & 20.50 & 24.30 & 27.90 & 28.20 & 28.40 & 28.50 \\
\hline Min & 26.60 & 25.20 & 24.20 & 23.20 & 18.20 & 18.30 & 18.20 & 20.30 & 24.30 & 27.70 & 28.20 & 28.40 \\
\hline Dissolved oxygen $(\mathrm{mg} / \mathrm{l}) \overline{\mathrm{x}}$ & 97.80 & 78.25 & 47.00 & 59.75 & 96.90 & 107.80 & 96.20 & 85.50 & 93.00 & 79.90 & 91.50 & 91.45 \\
\hline s & 0.98 & 0.77 & 1.27 & 0.21 & 0.28 & 1.06 & 0.42 & 1.34 & 0.28 & 0.56 & 2.12 & 0.07 \\
\hline Max & 98.50 & 78.80 & 47.90 & 59.90 & 97.10 & 108.60 & 96.50 & 86.50 & 93.20 & 80.30 & 93.00 & 92.00 \\
\hline Min & 97.10 & 77.70 & 46.10 & 59.60 & 96.70 & 107.10 & 95.90 & 84.60 & 92.80 & 79.50 & 90.00 & 90.90 \\
\hline Electrical conductivity $(\mu \mathrm{S} / \mathrm{cm})$ & 41.50 & 41.50 & 38.00 & 47.00 & 51.50 & 44.00 & 41.00 & 42.00 & 44.50 & 43.00 & 46.00 & 45.50 \\
\hline s & 0.70 & 0.70 & 0.00 & 2.82 & 0.70 & 1.41 & 0.00 & 0.00 & 0.70 & 0.00 & 0.00 & 0.70 \\
\hline Max & 42.00 & 42.00 & 38.00 & 49.00 & 52.00 & 45.00 & 41.00 & 42.00 & 45.00 & 43.00 & 46.00 & 46.00 \\
\hline Min_ & 41.00 & 41.00 & 38.00 & 45.00 & 51.00 & 43.00 & 41.00 & 42.00 & 44.00 & 43.00 & 46.00 & 45.00 \\
\hline $\mathrm{pH} \bar{x}$ & 6.45 & 6.50 & 645 & 6.25 & 6.90 & 6.75 & 7.15 & 7.00 & 7.00 & 6.90 & 7.10 & 7.10 \\
\hline s & 0.07 & 0.00 & 0.21 & 0.07 & 0.00 & 0.07 & 0.07 & 0.00 & 0.00 & 0.00 & 0.00 & 0.00 \\
\hline Max & 6.50 & 6.50 & 6.60 & 6.30 & 6.90 & 6.80 & 7.20 & 7.00 & 7.00 & 6.90 & 7.10 & 7.10 \\
\hline Min & 6.40 & 6.50 & 6.30 & 6.20 & 6.90 & 6.70 & 7.10 & 7.00 & 7.00 & 6.90 & 7.10 & 7.10 \\
\hline Chlorophyll -a $(\mu \mathrm{g} / \mid) \bar{x}$ & 0.00 & 6.55 & 0.00 & 1.09 & 1.63 & 0.00 & 1.09 & 1.76 & 2.45 & 1.21 & 3.18 & 1.09 \\
\hline $\mathrm{s}$ & 0.00 & 0.00 & 0.00 & 0.00 & 0.77 & 0.00 & 0.00 & 0.94 & 0.38 & 0.00 & 0.64 & 0.00 \\
\hline Max & 0.00 & 6.55 & 0.00 & 1.09 & 2.18 & 0.00 & 1.09 & 2.43 & 2.73 & 1.21 & 3.64 & 1.09 \\
\hline Min & 0.00 & 6.55 & 0.00 & 1.09 & 1.09 & 0.00 & 1.09 & 1.09 & 2.18 & 1.21 & 2.73 & 1.09 \\
\hline
\end{tabular}

\section{RESULTS}

\section{Water level}

The fluctuations in the water level of the Paraná River are shown in figure 2. The hydrological cycle had two phases: high water period (March 1992 to May 1992 and October 1992 to February 1993) and low water period (June 1992 to September 1992).

\section{Composition}

Table III lists rotifers recorded, including their constancy and known geographic distributions. Were identified 96 rotifer species belonging to 21 families, of which the most representative were: Brachionidae (20 species), Lecanidae (18 
species), and Trichoceridae (15 species). The most representative genera were Lecane (18 species), Trichocerca (15 species), and Brachionus (11 species). Among the bdelloids, which include periphytic and benthic species, were identified two species: Dissotrocha aculeata Ehrenberg, 1832 and D. macrodactyla (Ehrenberg, 1838) (Philodinidae).

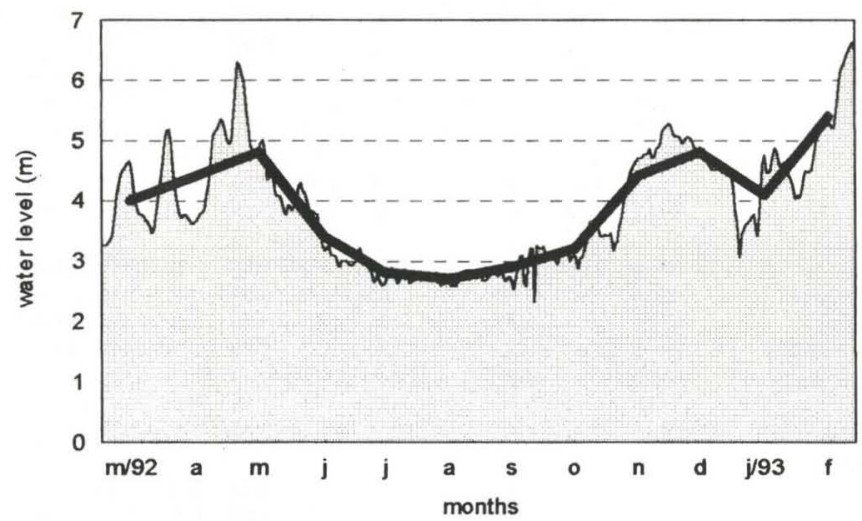

Fig. 2. Water level (m) of the Paraná River measured between March 1992 and February 1993. Dotted line $=$ average and solid area $=$ daily measuraments .

Table II. Densities of the principal species (ind $/ \mathrm{m}^{3}$ ) collected at each sampling station. These data were used in the PCA.

\begin{tabular}{|c|c|c|c|c|c|c|c|c|c|c|c|c|}
\hline Species & Mar & Apr & May & Jun & Jul & Aug & Sep & Oct & Nov & Dec & Jan & Feb \\
\hline \multicolumn{13}{|l|}{ Station 1} \\
\hline L. bulla & 327 & 1097 & 336 & 147 & 181 & 0 & 0 & 1287 & 758 & 13117 & 3767 & 8150 \\
\hline L. curvicornis & 903 & 10333 & 47 & 26 & 38 & 0 & 28 & 148 & 2667 & 12867 & 7633 & 4167 \\
\hline L. luna & 35 & 56 & 5 & 0 & 0 & 0 & 0 & 0 & 0 & 0 & 0 & 0 \\
\hline L. elsa & 25 & 917 & 2 & 0 & 5 & 0 & 0 & 69 & 0 & 550 & 733 & 2467 \\
\hline L. leontina & 36 & 111 & 68 & 9 & 2 & 0 & 0 & 0 & 33 & 300 & 100 & 100 \\
\hline L. papuana & 63 & 28 & 0 & 0 & 0 & 0 & 0 & 0 & 33 & 617 & 2683 & 50 \\
\hline D. p. propatula & 54 & 1306 & 0 & 7 & 31 & 0 & 28 & 74 & 633 & 1183 & 3000 & 817 \\
\hline P. p. patulus & 125 & 2306 & 10 & 9 & 9 & 0 & 0 & 2009 & 117 & 67 & 17 & 750 \\
\hline P. q. quadricornis & 204 & 694 & 38 & 20 & 7 & 0 & 9 & 148 & 167 & 5517 & 217 & 1400 \\
\hline T. pusilla & 0 & 0 & 0 & 6 & 27 & 1630 & 3000 & 56 & 17 & 17 & 50 & 0 \\
\hline F. terminalis & 21 & 917 & 56 & 0 & 2 & 111 & 185 & 1324 & 117 & 50 & 450 & 600 \\
\hline B. calyciflorus & 1 & 0 & 5 & 0 & 0 & 2512 & 1537 & 56 & 17 & 17 & 33 & 0 \\
\hline K. tropica & 16 & 0 & 108 & 23 & 61 & 923 & 4685 & 250 & 100 & 50 & 150 & 0 \\
\hline T. patina & 121 & 750 & 17 & 0 & 2 & 0 & 28 & 19 & 100 & 83 & 1133 & 550 \\
\hline Bdelloidea & 488 & 1028 & 65 & 77 & 54 & 0 & 102 & 713 & 333 & 767 & 3050 & 783 \\
\hline \multicolumn{13}{|l|}{ Station 2} \\
\hline L. bulla & 1639 & 3000 & 1370 & 361 & 3037 & 556 & 500 & 833 & 53 & & 625 & 9650 \\
\hline L. curvicornis & 28 & 7167 & 74 & 181 & 333 & 0 & 0 & 0 & 825 & 9300 & 1088 & 9700 \\
\hline L. Iuna & 222 & 0 & 0 & 0 & 37 & 0 & 111 & 111 & 0 & 0 & 0 & 0 \\
\hline L. elsa & 0 & 167 & 0 & 0 & 0 & 0 & 0 & 0 & 0 & 14000 & 38 & 1650 \\
\hline L. leontina & 333 & 333 & 593 & 14 & 148 & 111 & 167 & 75 & 1150 & 63 & 1050 & 93 \\
\hline L. papuana & 0 & 0 & 0 & 0 & 0 & 0 & 0 & 0 & 125 & 0 & 38 & 950 \\
\hline D. p. propatula & 28 & 750 & 37 & 14 & 111 & 0 & 56 & 0 & 0 & 300 & 25 & 1000 \\
\hline P. p. patulus & 0 & 0 & 444 & 0 & 0 & 0 & 111 & 111 & 500 & 100 & 75 & 1200 \\
\hline P. q. quadricornis & 167 & 83 & 297 & 97 & 148 & 0 & 56 & 56 & 0 & 2500 & 0 & 1150 \\
\hline T. pusilla & 0 & 0 & 0 & 0 & 0 & 0 & 0 & 0 & 0 & 0 & 0 & 0 \\
\hline
\end{tabular}


Table II. Continued.

\begin{tabular}{|c|c|c|c|c|c|c|c|c|c|c|c|c|}
\hline Species & Mar & Apr & May & Jun & Jul & Aug & Sep & Oct & Nov & Dec & Jan & Feb \\
\hline F. terminalis & 0 & 0 & 0 & 0 & 0 & 0 & 1056 & 556 & 0 & 0 & 0 & 1650 \\
\hline B. calyciflorus & 0 & 0 & 0 & 0 & 0 & 0 & 2000 & 1389 & 1111 & 0 & 0 & 0 \\
\hline K. tropica & 0 & 0 & 37 & 0 & 0 & 38444 & 600 & 5278 & 0 & 0 & 0 & 0 \\
\hline T. patina & 0 & 833 & 630 & 14 & 667 & 333 & 56 & 0 & 0 & 150 & 88 & 750 \\
\hline Bdelloidea & 139 & 1833 & 704 & 0 & 630 & 333 & 444 & 0 & 250 & 1200 & 125 & 850 \\
\hline \multicolumn{13}{|l|}{ Station 3} \\
\hline L. bulla & 528 & 1100 & 2177 & 127 & 262 & 28 & 194 & 753 & 599 & 9133 & 4375 & 1300 \\
\hline L. curvicornis & 1343 & 1156 & 28 & 64 & 76 & 32 & 1851 & 90 & 235 & 1717 & 7467 & 1792 \\
\hline L. luna & 153 & 0 & 19 & 74 & 3 & 5 & 0 & 37 & 28 & 1117 & 0 & 0 \\
\hline L. e/sa & 51 & 239 & 4 & 4 & 8 & 0 & 6 & 0 & 23 & 167 & 383 & 200 \\
\hline L. leontina & 93 & 89 & 230 & 1 & 3 & 0 & 0 & 19 & 54 & 617 & 167 & 50 \\
\hline L. papuana & 23 & 6 & 0 & 2 & 0 & 0 & 0 & 0 & 3 & 242 & 1192 & 633 \\
\hline D.p.propatula & 130 & 191 & 0 & 27 & 113 & 0 & 0 & 31 & 57 & 825 & 808 & 15676 \\
\hline P. p.patulus & 583 & 1042 & 28 & 3 & 38 & 0 & 5 & 479 & 174 & 583 & 4166 & 275 \\
\hline P. q. quadricornis & 204 & 178 & 39 & 23 & 30 & 9 & 0 & 772 & 34 & 1892 & 200 & 408 \\
\hline T. pusilla & 0 & 0 & 0 & 23 & 758 & 1167 & 0 & 0 & 0 & 0 & 0 & 0 \\
\hline F. terminalis & 102 & 117 & 100 & 16 & 0 & 116 & 125 & 926 & 19 & 17 & 1333 & 1233 \\
\hline B. calyciflorus & 5 & 0 & 0 & 1 & 4 & 258 & 560 & 0 & 6 & 17 & 0 & 0 \\
\hline K. tropica & 144 & 14 & 96 & 10 & 8 & 375 & 2744 & 191 & 17 & 0 & 0 & 0 \\
\hline T. patina & 264 & 183 & 76 & 44 & 63 & 5 & 23 & 34 & 66 & 208 & 450 & 125 \\
\hline Bdelloidea & 482 & 139 & 127 & 76 & 54 & 111 & 120 & 228 & 173 & 275 & 1100 & 500 \\
\hline \multicolumn{13}{|l|}{ Station 4} \\
\hline L. bulla & 142 & 172 & 111 & 33 & 34 & 25 & 56 & 611 & 433 & 7850 & 850 & 2050 \\
\hline L. curvicornis & 258 & 44 & 0 & 30 & 158 & 30 & 29 & 0 & 67 & 50 & 50 & 75 \\
\hline L. luna & 8 & 0 & 0 & 0 & 0 & 0 & 7 & 56 & 0 & 1050 & 917 & 1625 \\
\hline L.elsa & 8 & 43 & 0 & 4 & 26 & 0 & 3 & 37 & 17 & 50 & 33 & 225 \\
\hline L. leontina & 17 & 39 & 111 & 0 & 21 & 0 & 0 & 20 & 167 & 500 & 83 & 125 \\
\hline L. papuana & 0 & 15 & 0 & 0 & 0 & 0 & 0 & 0 & 0 & 100 & 67 & 825 \\
\hline D. p. propatula & 33 & 0 & 0 & 0 & 0 & 20 & 10 & 19 & 33 & 50 & 117 & 0 \\
\hline P. p. patulus & 8 & 83 & 56 & 15 & 4 & 0 & 7 & 74 & 250 & 150 & 150 & 200 \\
\hline P. q. quadricornis & 75 & 103 & 167 & 59 & 30 & 46 & 26 & 37 & 33 & 100 & 33 & 300 \\
\hline T. pusilla & 0 & 0 & 0 & 0 & 0 & 0 & 7 & 0 & 0 & 0 & 0 & 0 \\
\hline F. terminalis & 42 & 20 & 42 & 11 & 9 & 0 & 10 & 129 & 0 & 0 & 17 & 0 \\
\hline B. calyciflorus & 25 & 5 & 0 & 0 & 43 & 51 & 13 & 19 & 0 & 0 & 0 & 0 \\
\hline K. tropica & 33 & 29 & 125 & 7 & 9 & 76 & 160 & 19 & 17 & 0 & 17 & 0 \\
\hline T. patina & 192 & 64 & 694 & 200 & 325 & 56 & 26 & 148 & 50 & 600 & 250 & 25 \\
\hline Bdelloidea & 233 & 29 & 13 & 37 & 30 & 71 & 33 & 148 & 100 & 800 & 317 & 350 \\
\hline \multicolumn{13}{|l|}{ Station 5} \\
\hline L. bulla & 290 & 640 & 264 & 147 & 80 & 69 & 146 & 1331 & 454 & 2000 & 275 & 268 \\
\hline L. curvicornis & 12 & 170 & 21 & 2 & 3 & 6 & 15 & 31 & 158 & 288 & 567 & 52 \\
\hline L. luna & 285 & 39 & 46 & 35 & 43 & 39 & 22 & 0 & 138 & 363 & 103 & 73 \\
\hline L. elsa & 5 & 10 & 0 & 0 & 0 & 0 & 0 & 22 & 25 & 75 & 30 & 0 \\
\hline L. leontina & 12 & 12 & 72 & 4 & 6 & 0 & 2 & 9 & 56 & 275 & 28 & 5 \\
\hline L. papuana & 24 & 10 & 9 & 0 & 0 & 0 & 0 & 9 & 0 & 25 & 33 & 12 \\
\hline D. p. propatula & 102 & 47 & 0 & 35 & 8 & 13 & 8 & 6 & 81 & 163 & 36 & 41 \\
\hline P. p. patulus & 24 & 19 & 12 & 0 & 6 & 0 & 29 & 17 & 54 & 25 & 125 & 89 \\
\hline P.q. quadricornis & 54 & 24 & 5 & 26 & 2 & 34 & 2 & 19 & 17 & 75 & 58 & 15 \\
\hline T.pusilla & 0 & 0 & 0 & 4 & 2 & 0 & 0 & 22 & 0 & 0 & 0 & 0 \\
\hline F. terminalis & 219 & 0 & 287 & 8 & 6 & 9 & 8 & 10 & 17 & 37 & 131 & 21 \\
\hline B. calyciflorus & 0 & 0 & 5 & 0 & 17 & 4 & 4 & 0 & 0 & 0 & 0 & 0 \\
\hline K. tropica & 26 & 6 & 61 & 32 & 7 & 73 & 64 & 18 & 202 & 75 & 25 & 0 \\
\hline T. patina & 146 & 194 & 141 & 54 & 12 & 35 & 9 & 0 & 48 & 75 & 64 & 0 \\
\hline Bdelloidea & 248 & 55 & 368 & 89 & 65 & 51 & 81 & 42 & 85 & 213 & 94 & 93 \\
\hline
\end{tabular}

The greatest number of species in the lake occurred in the open water region (72 species), and 61 species were found in the littoral region. Similarly in the river, 85 species were recorded in open water, and 67 species in the littoral. 82 species were identified in the connecting channel between the river and the lake. 
The Constancy Index suggested that a larger number of constant species were present in the river, chiefly in the littoral region, than in the channel, followed by the lake (Tab. III). Only eight species were constant at the five sampling stations: Dipleuchlanis propatula propatula (Gosse, 1886), Lecane curvicornis (Murray, 1913), L. leontina (Turner, 1892), L. bulla (Gosse, 1851), Plationus patulus patulus (Müller, 1786), Platyias quadricornis quadricornis (Ehrenberg, 1832), Testudinella patina (Hermann, 1783) and T. mucronata hauriensis (Gillard, 1967).

Over all, 55 species are of widespread distribution, 13 are pantropical, and 16 neotropical. Of these, 8 are endemic to South America (Tab. III). Trichocerca gracilis (Tessin, 1890) is a new record for the floodplain of the Upper Paraná River.

The greatest diversity of species was recorded at all stations in the high water period (Fig. 3).
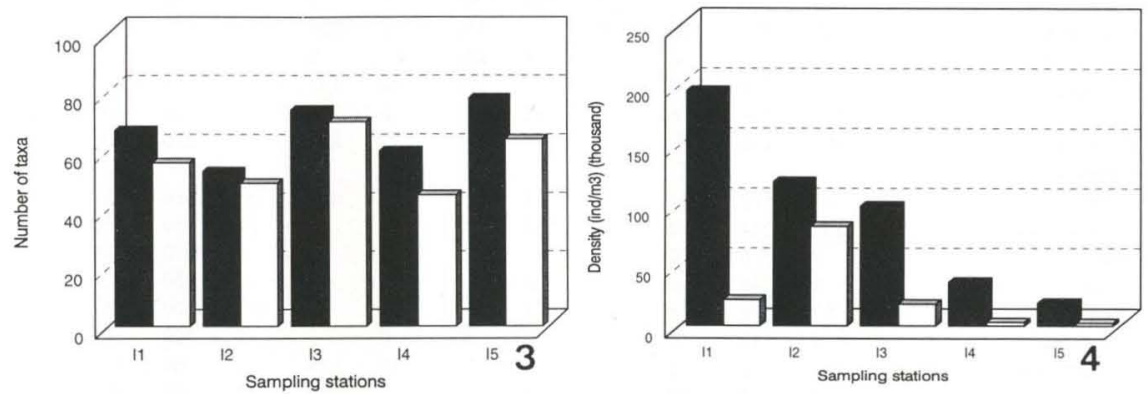

Figs 3-4. (3) Number of species in each period of the hydrolical cycle by sampling station; (4) density of rotifers (thousand ind $/ \mathrm{m}^{3}$ ) in each period of the hydrolical cycle by sampling station.

Low water, $\mathbf{a}$ high water.

\section{Density}

Comparing the total rotifer densities at the five sampling stations, considering only the mean of the stations with more than one collection depth, the greatest abundance occurred in the lake, followed by the channel, and then the river. Rotifer densities fluctuated from month to month at each station, with higher densities during the high water period (Fig. 4).

The most numerous species, in the whole system were Lecane bulla, $L$. curvicornis, L. luna (Müller, 1776), L. elsa Hauer, 1931, L. leontina, L. papuana (Murray, 1913), Dipleuchlanis propatula propatula, Keratella tropica (Apstein, 1907), Plationus patulus patulus, Platyias quadricornis quadricornis, Brachionus calyciflorus Pallas, 1776, Trichocerca pusilla (Lauterborn, 1898), Testudinella patina, and Filinia of terminalis (Plate, 1886). Bdelloids were numerous at all stations (Tab. II). All these species were considered for the PCA.

\section{Principal components analysis}

The results obtained by PCA showed that the principal components (CPI and CPII) explained $60.42 \%$ of the total variability of the data. 
Table III. Occurrence of species, Constancy Index and geographical distribution (Gd) in Patos Lake and the Ivinheima River between March 1992 and February 1993. (Wd) Widespread distribution, (Trop/p) pantropical, (Trop/n) neotropical and (end) endemic to South America (Koste 1978; Jose de PAGgI 1990; Bonecker et al. 1994). (C) Constant, (A) Accesory, (Ac) Accidental.

\begin{tabular}{|c|c|c|c|c|c|c|}
\hline \multirow{2}{*}{ Species } & \multicolumn{6}{|c|}{ Sampling stations } \\
\hline & 1 & 2 & 3 & 4 & 5 & GD \\
\hline \multicolumn{7}{|l|}{ Monogononta } \\
\hline Brachionus angularis Gosse, 1851 & & & $\mathrm{Ac}$ & & Ac & Wd \\
\hline B. budapestinesis Daday, 1885 & & & $A C$ & & & Wd \\
\hline B. dolabratus Harring, 1915 & $A C$ & & & $A C$ & $A C$ & Trop/n \\
\hline B. calyciflorus Pallas, 1766 & A & $A C$ & A & $A$ & $\mathrm{AC}$ & Wd \\
\hline B. falcatus Zacharias, 1898 & $A c$ & $A C$ & A & $\mathrm{C}$ & Ac & Wd \\
\hline B. quadridentatus quadridentatus Hermann, 1783 & $A c$ & $A c$ & A & $A c$ & $\mathrm{Ac}$ & Wd \\
\hline B. q. mirabilis (Daday, 1897) & $A C$ & & $A C$ & $A C$ & $A c$ & Tro/p \\
\hline B. mirus Daday, 1905 & $A C$ & & $A$ & $A C$ & A & Trop/n, end \\
\hline B. urceolaris (Müller, 1773) & $A$ & $A C$ & A & $A C$ & $A C$ & Wd \\
\hline B. caudatus Barrois \& Daday, 1884 & $A C$ & $A C$ & $A C$ & $\mathrm{C}$ & Ac & Trop/p (?) \\
\hline B. caudatus v. personatus Ahlstrom, 1940 & & & & & $\mathrm{Ac}$ & Wd \\
\hline Keratella americana Carlin, 1943 & $\mathrm{Ac}$ & $A c$ & $A c$ & C & A & Trop/p \\
\hline K. cochlearis Gosse, 1851 & $A$ & $\mathrm{Ac}$ & $A$ & C & A & Wd \\
\hline K. tropica (Apstein, 1907) & $\mathrm{C}$ & A & C & C & C & Trop/n \\
\hline K. Ienzi (Hauer, 1853) & $A C$ & $A C$ & $A C$ & C & A & Trop/n \\
\hline Plationus patulus patulus (Müller, 1786) & $\mathrm{C}$ & $\mathrm{C}$ & C & C & $\mathrm{C}$ & Wd \\
\hline P. macracanthus (Daday, 1905) & A & A & $\mathrm{C}$ & $\mathrm{C}$ & $\mathrm{C}$ & Trop/n \\
\hline Platyias quadricornis quadricornis (Ehrenberg, 1832) & $\mathrm{C}$ & $\mathrm{C}$ & $\mathrm{C}$ & $\mathrm{C}$ & $\mathrm{C}$ & Wd \\
\hline P. q. brevespinus (Daday, 1905) & A & Ac & A & A & $\mathrm{C}$ & Wd \\
\hline P. leloupi Gillard, 1957 & $A c$ & & $\mathrm{Ac}$ & & $A c$ & Trop/n \\
\hline \multicolumn{7}{|l|}{ Lecanidade } \\
\hline Lecane monostyla (Daday, 1897) & $A C$ & $A C$ & $A C$ & $A C$ & $A c$ & Trop/p \\
\hline L. quadridentata (Ehrenberg, 1832) & $A C$ & $\mathrm{Ac}$ & $A C$ & $A C$ & $A$ & Trop/p \\
\hline L. amazonica (Murray, 1913) & $A c$ & & $A C$ & $A C$ & $A C$ & Trop/n, end \\
\hline L. closterocerca (Schmarda, 1859) & $A$ & $\mathrm{Ac}$ & A & $A c$ & $A$ & Trop/p \\
\hline L. bulla (Gosse, 1851) & C & C & C & C & C & Wd \\
\hline L. lunaris (Ehrenberg, 1832) & $A C$ & A & A & C & $\mathrm{C}$ & Wd \\
\hline L. cornuta (Müller, 1786) & $A$ & $\mathrm{C}$ & $A C$ & C & A & Wd \\
\hline L. papuana (Murray, 1913) & A & $\mathrm{Ac}$ & A & A & A & Trop/p \\
\hline L. curvicornis (Murray, 1913) & $\mathrm{C}$ & $\mathrm{C}$ & $\mathrm{C}$ & $\mathrm{C}$ & C & Trop/p \\
\hline L. leontina (Turner, 1892) & $\mathrm{C}$ & $\mathrm{C}$ & C & C & C & Trop/p \\
\hline L. elsa Hauer, 1931 & $A$ & A & A & C & $A$ & Wd \\
\hline L. ludwigi (Eckstein, 1883) & $A C$ & $\mathrm{C}$ & A & $\mathrm{C}$ & C & Trop/p \\
\hline L. proiecta Hauer, 1956 & $A C$ & & $A C$ & $A C$ & $A C$ & Trop/n, end \\
\hline L. luna (Maller, 1776) & $A$ & A & A & $A$ & $\mathrm{C}$ & Wd \\
\hline L. stichaea Harring, 1913 & $A C$ & A & A & & $A C$ & Wd \\
\hline L. signifera (Jennings, 1886) & $A C$ & & $\mathrm{Ac}$ & & Ac & Wd \\
\hline L. aculeata (Jakubski, 1912) & & $A c$ & & & $A C$ & Trop \\
\hline Lecane sp. & & $A C$ & & & $A c$ & \\
\hline \multicolumn{7}{|l|}{ Trichocercidae } \\
\hline Trichocerca (Diurella) bidens (Lucks, 1912) & & & & & $A C$ & Wd \\
\hline T. (D) similis (Wierzejski, 1893) & & & $A C$ & & Ac & Wd \\
\hline T. (D) similis grandis (Hauer, 1965) & & & $\mathrm{Ac}$ & Ac & & Wd \\
\hline T. (D) insignis (Herrick, 1885) & & & & $A c$ & & Wd? \\
\hline T. (D) porcellus (Gosse, 1886) & $\mathrm{Ac}$ & & $\mathrm{Ac}$ & $A c$ & $A c$ & Wd \\
\hline T. cylindrica chattoni De Beauchamp, 1907 & $A c$ & $A C$ & & $A$ & A & Wd \\
\hline T. bicristata Gosse, 1887 & $A c$ & $A C$ & A & $A C$ & $\mathrm{C}$ & Wd \\
\hline T. gracilis (Tessin, 1890) & $A C$ & $A C$ & & $A c$ & $A c$ & Wd \\
\hline T. scipio Pejler, 1962 & & & $\mathrm{Ac}$ & $\mathrm{Ac}$ & & $W d$ \\
\hline T. capucina Wierzejski \& Zacharias, 1893 & & & $\mathrm{Ac}$ & & $A c$ & Wd \\
\hline T, elongata (Gosse, 1886) & $A c$ & $A C$ & $A c$ & $A C$ & $A$ & Wd \\
\hline T. pusilla (Lauterborn, 1898) & $A$ & & $A C$ & $A C$ & A & Wd \\
\hline Trichocerca sp 1 & $A C$ & $A C$ & $\mathrm{AC}$ & $A C$ & $A c$ & \\
\hline Trichocerca sp 2 & & & $A C$ & $A C$ & $\mathrm{Ac}$ & \\
\hline Trichocerca sp 3 & $A C$ & & & & & \\
\hline
\end{tabular}


Table III. Continued. (C) Constant, (A) Accesory, (Ac) Accidental.

\begin{tabular}{|c|c|c|c|c|c|c|}
\hline \multirow{2}{*}{ Species } & \multicolumn{6}{|c|}{ Sampling stations } \\
\hline & 1 & 2 & 3 & 4 & 5 & GD \\
\hline \multicolumn{7}{|l|}{ Euchlanidae } \\
\hline Euchlanis dilatata Ehrenberg, 1832 & Ac & Ac & Ac & A & $\mathrm{Ac}$ & Wd \\
\hline E. incisa Carlin, 1939 & & Ac & $A c$ & & $A C$ & Wd \\
\hline Dipleuchlanis propatula propatula (Gosse, 1886) & C & C & C & C & C & Wd \\
\hline D. propatula f. macrodactyla (Hauer, 1965) & $A c$ & A & $A c$ & & $A C$ & Trop/n, end \\
\hline Manfredium eudactylotum (Gosse, 1886) & $A c$ & Ac & A & Ac & A & Wd \\
\hline \multicolumn{7}{|l|}{ Mytilinidae } \\
\hline Mytilina ventralis (Ehrenberg, 1832) & A & C & A & C & $\mathrm{C}$ & Wd \\
\hline M. acanthophora Hauer, 1938 & Ac & & Ac & & $\mathrm{Ac}$ & Trop/n, end \\
\hline M. macrocera (Jennings, 1894) & $A C$ & Ac & $A c$ & Ac & & Trop/n \\
\hline \multicolumn{7}{|l|}{ Synchaetidae } \\
\hline Synchaeta stylata Wierzejski, 1893 & & & $A c$ & & & Wd \\
\hline Synchaeta sp. & $A C$ & Ac & $A C$ & & $A c$ & \\
\hline Polyarthra vulgaris Carlin, 1943 & C & A & $\mathrm{C}$ & A & A & $W d$ \\
\hline P. remata (Skorikov, 1896) & $A c$ & $A c$ & $A c$ & & $A c$ & Wd \\
\hline Ploesoma truncata (Levander, 1894) & & $A c$ & $A c$ & $\mathrm{Ac}$ & $A c$ & Wd \\
\hline \multicolumn{7}{|l|}{ Testudinellidae } \\
\hline Testudinella patina (Hermann, 1783) & C & C & C & C & C & Wd \\
\hline T. mucronata hauriensis (Gillard, 1967) & C & C & C & C & C & Trop/n, end \\
\hline T. tridentata amazonica Thomasson, 1971 & & & & & Ac & Wd \\
\hline T. ohlei Koste, 1972 & & & & & Ac & Trop/n, end \\
\hline T. ahlstromi (Hauer, 1956) & & & & & $A C$ & Trop/n, end \\
\hline \multicolumn{7}{|l|}{ Conochilidae } \\
\hline Conochilus unicornis Rousselet, 1892 & C & A & A & A & A & Wd \\
\hline C. coenobasis Skorikov, 1914 & $A C$ & & $\mathrm{Ac}$ & & $A c$ & Wd \\
\hline C. natans (Seligo, 1900) & & & Ac & & $\mathrm{Ac}$ & Wd \\
\hline C. dossuarius (Hudson, 1875) & & & $\mathrm{Ac}$ & $A c$ & $A C$ & Wd \\
\hline \multicolumn{7}{|l|}{ Filiniidae } \\
\hline Filinia pejleri Hutchinson, 1964 & & & $A c$ & & & Trop/p \\
\hline F. longiseta (Ehrenberg, 1834) & C & Ac & $\mathrm{C}$ & A & A & Wd \\
\hline F. saltator (Gosse, 1886) & A & $A C$ & C & Ac & C & Trop/n \\
\hline F. cf terminalis (Plate, 1886) & $\mathrm{C}$ & $\mathrm{Ac}$ & $\mathrm{C}$ & $\mathrm{C}$ & C & Wd \\
\hline F. opoliensis (Zacharias, 1891) & $A c$ & $A c$ & Ac & $A C$ & $A C$ & Wd \\
\hline \multicolumn{7}{|l|}{ Hexarthridae } \\
\hline Hexarthra intermedia braziliensis (Haver, 1953) & Ac & & $A c$ & $A c$ & $A c$ & Trop/n \\
\hline H. mira (Hudson, 1871) & & & & & $A C$ & Wd \\
\hline \multicolumn{7}{|l|}{ Trichotriidae } \\
\hline Trichotria tetractis (Ehrenberg, 1830) & A & C & A & C & C & Wd \\
\hline Macrochaetus sericus (Thorpe, 1893) & Ac & $A c$ & Ac & $A c$ & A & Trop/p \\
\hline \multicolumn{7}{|l|}{ Colurellidae } \\
\hline Lepadella ovalis (Müler, 1786) & A & C & A & $\mathrm{C}$ & C & Wd \\
\hline L. benjamini Harring, 1916 & A & $A c$ & A & Ac & Ac & Trop/p \\
\hline \multicolumn{7}{|l|}{ Epiphanidae } \\
\hline Epiphanes clavulata (Ehrenberg, 1832) & & & $A c$ & & $A c$ & Wd \\
\hline \multicolumn{7}{|l|}{ Asplanchnidae } \\
\hline Asplanchna (A) sieboldi (Leydig, 1854) & C & & C & $A C$ & $A c$ & Wd \\
\hline \multicolumn{7}{|l|}{ Proalidae } \\
\hline Proales sp. & $\mathrm{Ac}$ & $A c$ & $A c$ & & & \\
\hline Notommatidae & & & & & & \\
\hline Cephalodella sp. & $A c$ & A & $A c$ & $A c$ & $\mathrm{Ac}$ & \\
\hline $\begin{array}{l}\text { Flosculariidae } \\
\text { Ptygura sp. }\end{array}$ & & & & & & \\
\hline $\begin{array}{l}\text { Ptygura sp. } \\
\text { Dicranophoridae }\end{array}$ & & $A C$ & $A c$ & & $A c$ & \\
\hline $\begin{array}{l}\text { Dicranophoridae } \\
\text { Dicranophorus sp. }\end{array}$ & & & & & & \\
\hline $\begin{array}{l}\text { Dicranophorus sp. } \\
\text { Gastropodidae }\end{array}$ & A & & A & Ac & Ac & \\
\hline Ascomorpha ecaudis (Perty, 1859) & Ac & $A C$ & $A C$ & A & Ac & Wd \\
\hline Collothecidae & & & & & & \\
\hline Collotheca sp. & $A c$ & & $A C$ & & & \\
\hline Bdelloidea & & & & & & \\
\hline Philodinidae & & & & & & \\
\hline Dissotrocha aculeata Ehrenberg, 1832 & Ac & A & A & Ac & Ac & Wd \\
\hline D. macrodactyla (Ehrenberg, 1838) & & & & $A c$ & & $?$ \\
\hline
\end{tabular}


Analyzing the structure coefficients and the location of the sampling units at CPI (38.54\%) and CPII (21.87\%) together, was found that the highest rotifer densities occurred in the lake $(a=1$ and $b=2)$, during the high water period ( $2=$ April, $9=$ November, $10=$ December, $11=$ January, $12=$ February), when the following limnological conditions predominated: high values of electrical conductivity and temperature; low concentrations of dissolved oxygen, and low $\mathrm{pH}$ values (CPI, solid line). Moreover, high rotifer densities also were associated with the higher concentrations of chlorophyll-a observed at the end of the low water period ( $7=$ September) and during the high water period ( $9=$ November and $11=$ January) (CPII, dotted line) (Fig. 5). The two principal components explained the spatial and temporal variations, where the CPI showed chiefly the influence of the water level and the CPII reflected the importance of chlorophyll-a. The sum of the three first principal components of PCA, employing the abiotic variables and the most abundant species, explained $58.58 \%$ of the total variability of the data.

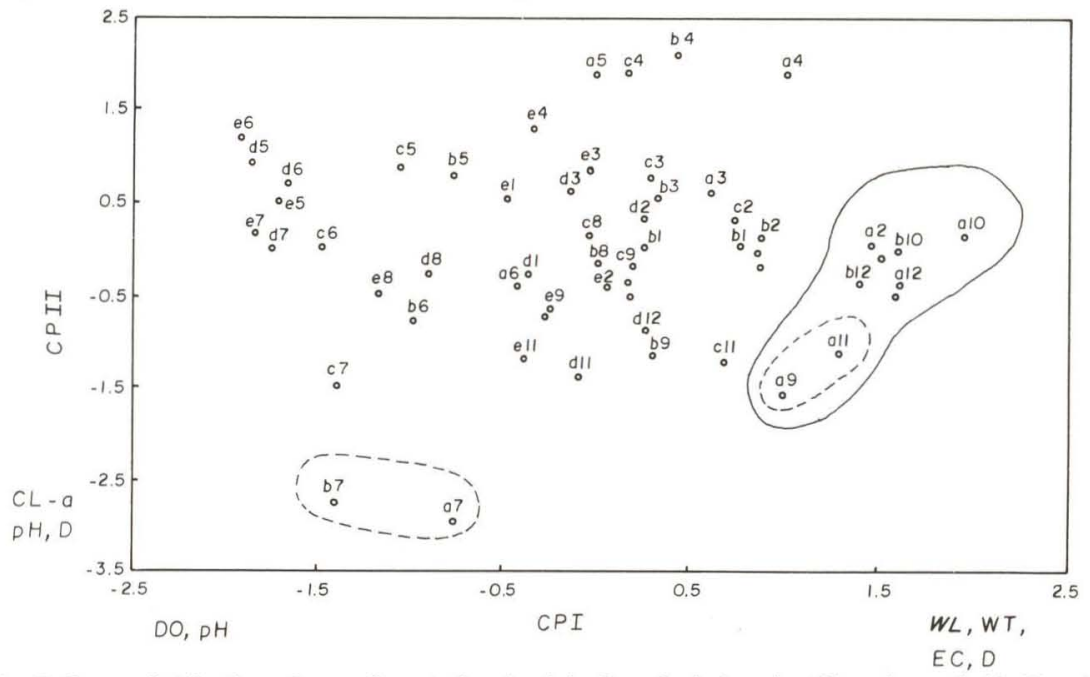

Fig. 5. Score distribution of sampling station ( $a=1, b=2, c=3, d=4$ and $e=5)$ and month ( $1=$ March, 2=April, 3=May, 4=June, 5=July, 6=August, $7=$ September, 8=October, 9=November, 10=December, $11=$ January and $12=$ February) among principal components defined by environmental variables. (-) First component, (- - -) second component.

The ordination of the stations and collection months along CPI (36.6\%) indicates that most of the species negatively correlated with this component were more abundant in the lake $(\mathrm{a}=1$ and $\mathrm{b}=2)$ and the channel $(\mathrm{a}=3)$, during the high water period ( $10=$ December, $11=$ January, $12=$ February) (solid line). These environments and collection months had high water levels, high temperature, and electrical conductivity values, and low concentrations of dissolved oxygen. Brachionus calyciflorus and Keratella tropica were more abundant in the open water region of the lake $(\mathrm{a}=\mathrm{I} 1)$ at the end of the low water period $(6=$ August and $7=$ September), when the limnological characteristics were the opposite of those described above (Fig. 6). 


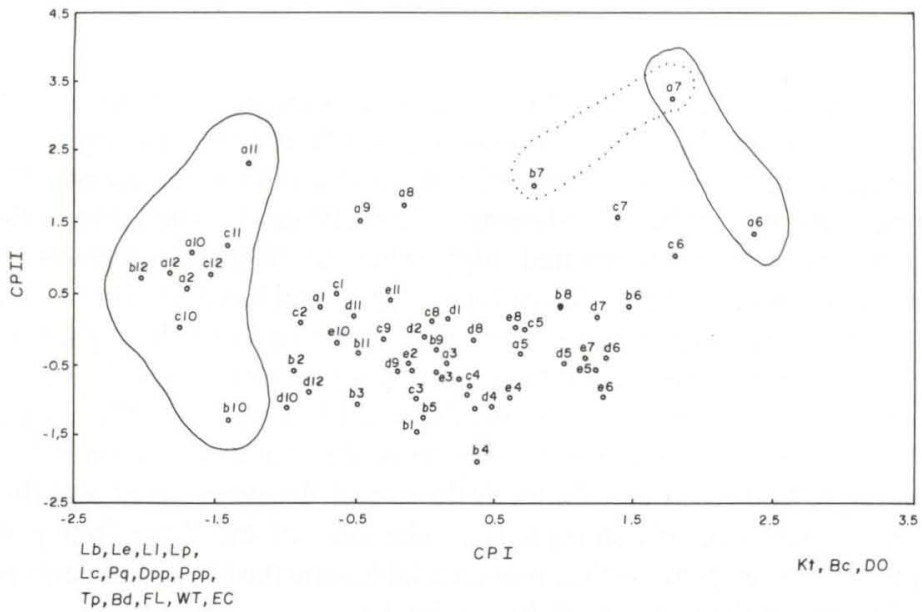

Fig. 6. Score distribution of sampling station $(a=1, b=2, c=3, d=4$ and $e=5)$ and month (1=March, 2=April, 3=May, 4=June, 5=July, 6=August, $7=$ September, $8=$ October, $9=$ November, 10=December, 11=January and 12=February) among principal components defined by the densities of the principal species and environmental variables. (-) First component, (- - -) second component.

CPII (11.8\%) showed that Brachionus calyciflorus, Filinia cf terminalis and Trichocerca pusilla predominated in the lake $(\mathrm{a}=1$ and $\mathrm{b}=2)$, and in month when higher chlorophyll-a concentrations occurred (7=September) (dotted line) (Fig. 7). According to the CPIII (10.71\%), in this environment T. pusilla was also important in the collection months with greater water transparency (4=June and 5=July) (dashed line), and Lecane luna in the month with high concentrations of dissolved oxygen and when the $\mathrm{pH}$ tended to the alkaline range $(7=$ September) (dashed line) (Fig. 7).

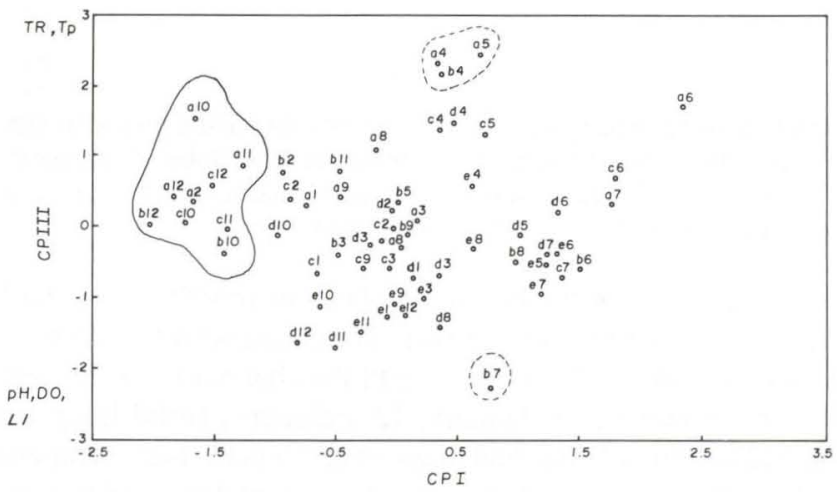

Fig. 7. Score distribution of sampling station $(a=1, b=2, c=3, d=4$ and $e=5)$ and month ( $1=$ March, 2=April, 3=May, 4=June, 5=July, 6=August, $7=$ September, $8=$ October, $9=$ November, 10=December, $11=$ January and $12=$ =February) among principal components defined by environmental variables. (-) third component. 


\section{DISCUSSION}

Of the 28 genera identified, Lecane, Brachionus, and Trichocerca showed the highest richness of species, making up almost half of the number of records (46.4\%). The richness of Lecane is well documented for tropical continental waters (Koste \& JosÉ De PAGgi 1982; JosÉ De PAGGI 1990; ZoPPI DE RoA et al. 1993; BONECKER et al. 1994; LANSAC-TÔHA et al. 1997). Brachionus is also more representative in the tropics (PEJLER 1977; FERNANDO 1980; DUMONT 1983).

More species were found in the open water regions of these systems. These results differ from those verified for Guaraná Lake, also located on this floodplain (BONECKER et al. 1994). Guaraná Lake is small with extensive aquatic macrophyte beds which favor greater habitat diversity. On the other hand, Patos Lake has a larger area, with less developed macrophyte beds. Another fact that may explain these results is that in the open water region of Patos Lake, sampling was performed at three depths of the water column, whereas in the littoral region it was only conducted at the surface.

The composition of the rotifer fauna was influenced by the variations in the water level of the Paraná River, with more species recorded during the high water period. For example, at that time, species characteristic of the littoral region such as Lecane signifera (Jennings, 1886), L. papuana, and L. proiecta (Hauer, 1956) occurred in the open water. The rise in water level permits greater exchange of fauna between the littoral and open water regions. This influence of the hydrological regime on the composition of the rotifers has been observed in other environments of the floodplains of the Upper Paraná (LANSAC-TÔHA et al. 1992, 1997; BONECKER et al. 1994; CAMPOS et al. 1996) and the Middle Paraná (JosÉ DE PAGGI 1988; PAGGI \& José DE PAGgi 1990), the Amazon basin (Koste \& RoBerTson 1983; KosTE et al. 1984; BozelLi 1992), and the Orinoco River (VÁSQUEZ 1984a).

Most of the rotifer species (57.3\%) have a widespread geographical distribution. Several species endemic to the neotropical region and even to South America were present. Most neotropical endemics were members of the Brachionidae (Brachionus dolabratus Harring, 1915 and Plationus macracanthus (Daday, 1905), among others), as in other environments of this floodplain (BONECKER et al. 1994; LANSAC-TÔHA et al. 1997) and the floodplain of the Middle Paraná River, Argentina (PAGGI \& José DE PAGGI 1990).

The highest rotifer densities occurred in the lake, as evidenced by the PCA. Greater abundances of rotifers in lentic habitats were also seen in other studies in the floodplain of the Upper Paraná River (BONECKER \& LANSAC-TÔHA 1996; LANSAC-TôHA et al. 1997), the Middle Paraná River (PAGGi \& José DE PAGgi 1990), and in the Amazon basin (BozelLI 1994).

In different aquatic environments subject to fluctuations in the water level, several studies have shown that greatest rotifer densities occur during the low water period (HARDY et al. 1984; VÁSQUEZ 1984b; VÁSQUEZ \& SANCHEZ 1984; PAGGI \& JosÉ DE PAGGI 1990; BozELLI 1994). The results of these studies contrast with ours, which recorded that the greatest abundances during high water, as illustrated by the PCA. This unusual occurrence may be related to the fact that the littoral 
region, during the high water period, is subject to strong mixing currents. During low water, in the open water of the lake and the channel typically planktonic species predominate: Keratella tropica, K. cochlearis Gosse, 1851, Brachionus calyciflorus, Trichocerca pusilla, and Conochilus unicornis Rousselet, 1892. In these same environments, during the high water, the most representative species were those typical of littoral regions, such as Lecane curvicornis, L. bulla, L. elsa, Plationus patulus patulus, Platyias quadricornis quadricornis, and Dipleuchlanis propatula propatula. This fact is shown by the PCA. According to FERNANDO (1980), opportunistic species typical of littoral regions may invade the open water region and become dominant temporarily.

The increment in the rotifer densities in the river during high water may also be caused by the contribution of fauna from the lake and other littoral lakes associated with the river. SAUNDERS \& LEWIS (1988) observed that highest rotifer densities in the Caura River, Venezuela, also during high water, could be the result of the incorporation of animals from lentic habitats of the floodplain. Completing this reasoning, changes in the water volume activate backwaters or isolated areas, transporting animals to the river populations (JosÉ DE PAGGI 1981). The higher densities during high water may also be explained by the fact that the rotifers are opportunistic organisms capable of producing large populations in unstable environmental conditions (ALLAN 1976).

The water level was not the only variable correlated with the seasonal fluctuations in the rotifer densities. PCA also showed that the highest total densities were correlated with high temperatures and lower concentrations of dissolved oxygen. For some species like Brachionus calyciflorus, Lecane luna and Keratella tropica, dissolved oxygen seems to be a limiting factor, as is pH. BÉRZINS \& PEJLER (1989) affirm that oxygen concentration is an important factor in the temporal distribution of the rotifers. They recorded the occurrence of Brachinous calyciflorus, for example, at $\mathrm{pH}$ between 7.0 and 10.0, with highest abundance near 8.0 (BÉRZINS \& PEJLER 1987). Was found this species more abundant at $\mathrm{pH}$ ca. 7.5. Temperature is also clearly important in the development of rotifer populations, especially for egg development, since the birth rate is different for each individual of the same species. HOFFMANN (1977) emphasized that temperature is very important for understanding changes in species abundance throughout the year.

Chlorophyll-a was also important in the seasonal variation of the total and specific rotifer densities, especially for Filinia cf terminalis, Brachionus calyciflorus, and Trichocerca pusilla. As the PCA showed, the greatest densities of these organisms were related to higher values of chlorophyll-a concentration. Most rotifers are omnivorous, filter-feeders, selecting food particles according to their size and quality (JosÉ DE PAGGI 1978). Brachionus calyciflorus has a wide feeding spectrum, being an active consumer of algae and bacteria (POURRIOT 1977; PEJLER 1983; ROTHHAUPT 1990).

Densities of some species are weakly correlated with concentrations of chlorophyll-a: Lecane elsa, L. curvicornis, L. leontina, Platyias quadricornis quadricornis, Dipleuchanis propatula propatula and Bdelloidea. These species are typical of littoral regions with aquatic vegetation. Phytoplankton may not be the 
main food of these species, which may consume preferentially detritus and/or bacteria that, according to WETZEL (1990) are very abundant in these regions because of the decomposition of aquatic macrophytes. Species of the genus Lecane are detritus and bacteria feeders, and $D$. propatula propatula is exclusively detritivorous (POURRIOT 1977).

Studies undertaken by BONECKER \& LANSAC-TÔHA (1996) in this floodplain also showed that the densities of the chief rotifer species were influenced mainly by chlorophyll-a, dissolved oxygen, the fluviometric level, and water temperature.

\begin{abstract}
ACKNOWLEDGEMENTS. We thank Dr. Janet W. Reid for assistence with the English text. Thanks are due to our colleagues Dr. Susana José de Paggi for the identification of some species; Dr. Sérgio Luiz Costa Bonecker, Dr. Sidinei Magela Thomaz, Luiz Felipe Machado Velho and Luis Maurício Bini for their suggestions and their help in data analysis; to the staff of the Center for Research in Limnology, Ichthyology and Aquaculture (Nupélia, Universidade Estadual de Maringá) for technical and scientific support; to PADCT/CIAMB for the necessary financial support, to CAPES for several scholarships grants.
\end{abstract}

\title{
REFERENCES
}

AlLAN, J.D. 1976. Life history patterns in zooplankton. Amer. Nat. 110 (971): 165-180.

BÉRzINS, B. \& B. PEJLER. 1987. Rotifer occurrence in relation to pH. Hydrobiologia 147: 107-116

1989. Rotifer occurrence in relation to oxygen content. Hydrobiologia 183: $165-172$.

BONECKER, C.C. \& F.A. LANSAC-TÔHA. 1996. Community structure of rotifers in two environments of the high river Paraná floodplain (MS), Brazil. Hydrobiologia 325 (2): 137-150.

BoneCKeR, C.C.; F.A LANSAC-TÔHA \& A. STAuB. 1994. Qualitative study of rotifers in different environments of the high Paraná floodplain (MS), Brazil. Rev. Unimar, Maringá, 16 (Supl. 3): 1-16.

Bozelli, R.L. 1992. Composition of the zooplankton community of Batata and Mussurá Lakes and of the Trombetas River, State of Pará, Brazil. Amazoniana 12 (2): 239-261.

1994. Zooplankton community density in relation to water level flutuations and inorganic turbidity in an Amazonian lake, "Lago Batata", State of Pará, Brazil. Amazoniana 13 (2): 17-32.

CAMPOS, J.R.C.; F.A. LANSAC-TÔHA; M.A. NUNES; A.P.P. GARCIA \& F.R. PRADO.

1996. Composição da comunidade zooplanctônica de três lagoas da ilha Porto Rico, na planície de inundação do alto rio Paraná. Acta Limnol. Brasil. 8: 183-194.

DAJOZ, R. 1973. Ecologia Geral. São Paulo, Vozes/Edusp, 474p.

DUMONT, J. 1983. Biogeography of rotifers. Hydrobiologia 104: 19-30.

FERNANDO, C.H. 1980. The freshwater zooplankton of Sri Lanka, with a discussion of tropical freshwater zooplankton composition. Int. Rev. ges. Hydrobiol. 65 (1): $85-125$. 
HARDY, E.R.; B. ROBERTSON \& W. Koste. 1984. About the relationship between the zooplankton and fluctuating water levels of Lago Camaleão, a central Amazonian varzea lake. Amazoniana 9 (1): 43-52.

HoFFMANN, W. 1977. The influence of abiotic envirommental factors on population dynamics in planktonic rotifers. Arch. Hydrobiol. Beih. Ergebn. Limnol. 8: 77-83.

JosÉ DE PAGGI, S. 1978. Introduccion al estudio de los rotiferos. Rev. Asoc. Cienc. Nat. Lit. 9: 19-49.

1981. Variaciones temporales y distribución horizontal del zooplancton en algunos cauces secundarios del rio Paraná Médio. Stud. Neot. Fauna Environ. 16: 185-199.

1988. Estudio sinóptico del zooplancton de los principales cauces y tributarios del valle aluvial del rio Paraná: Tramo Goya-Diamante (II Parte). Stud. Neot. Fauna Environ. 23: 149-163.

1990. Ecological and biogeographical remarks on the rotifer fauna of Argentina. Rev. Hydrobiol. Trop. 23 (4): 297-311.

JunK, W.J.; P.B. BAyley \& R.E. SPARKS. 1989. The flood pulse concept in river-floodplain systems. Can. Spec. Publ. Fish. Aquat. Sci. 106: 110-127.

Koste, W. 1978. Rotatoria Die Rädertiere Mitteleuropas bergründet von Max Voigt-Monogononta. 2. Auflage neubearbeitet von Walter Koste. Berlin, Gebrüder Borntraeger, 673p.

Koste, W. \& S. JosÉ DE PAGGi. 1982. Rotifera of the Superorder Monogononta recorded from Neotropics. Gewäss. Abwäss. 68/69: 71-102.

Koste, W. \& B. RoberTSON. 1983. Taxonomic studies of the Rotifera (Phylum Aschelminthes) from a Central Amazonian várzea lake, Lago Camaleão (Ilha Machantaria, Rio Solimões, Amazonas, Brazil). Amazoniana 8 (2): 225-254.

Koste, W.; B. ROBERTSON \& E. HARDY. 1984. Further taxonomical studies of the Rotifera from Lago Camaleão, a Central Amazonian várzea lake (Ilha Marchantaria, Rio Solimões, Amazonas, Brazil). Amazoniana 8 (4): 555-576.

LANSAC-TÔHA, F.A.; A.F. LiMA; S.M. THOMAZ \& M.C. RoBerTo. 1992. Zooplâncton de uma planície de inundação do rio Paraná. I. Análise qualitativa e estrutura da comunidade. Rev. Unimar, Maringá, 14 (Supl): 35-55.

LANSAC-TÔHA, F.A.; C.C. BONECKER; L.F.M. VELHO \& A.F. LIMA. 1997. Zooplâncton do alto rio Paraná, p.117-155. In: A.E.A.M. VAzzoler; A.A. AGosTINHO \& N.S. HAHN (Eds). A Planície de Inundação do Alto Rio Paraná: aspectos físico-químicos, biológicos e sócio-econômicos. Maringá, EDUEM, 460p.

PAGGI, J.C. \& S. JosÉ DE PAGGI. 1990. Zooplâncton de ambientes lóticos e lênticos do rio Paraná médio. Acta Limnol. Brasil. 3: 685-719.

PEJLER, B. 1977. General problems on rotifer taxonomy and global distribution. Arch. Hydrobiol. 8: 212-220.

1983. Zooplanktonic indicators of trophy and their food. Hydrobiologia 101: 111-114.

PLA, L.E. 1986. Analisis multivariado: metodo de componentes principales. Washington, Organización de los Estados Americanos, 94p.

Pourriot, R. 1977. Food and feeding habits of Rotifera. Arch. Hydrobiol. Beih. 
Ergebn. Limnol. 8: 243-260.

ROBERTSON, B. \& E.R. HARDY. 1984. Zooplankton of Amazonian lakes and rivers, p.337-352. In: H. SiOLI (Ed.). The Amazon: limnology and landscape ecology of mighty tropical river and its basin. Dordrecht, W. Junk Publishers, Monographie Biological, vol. 56, 763p.

RoTHHAUPT, O.K. 1990. Changes of the functional responses of the rotifers Brachionus rubens and Brachionus calyciflorus with particle sizes. Limnol. Oceanogr. 35 (1): 24-32.

SAUNDERS, J.F. \& W.M. LEWIS. 1988. Zooplankton abundance in the Caura River, Venezuela. Biotropica 20 (3): 206-214.

SEgERs, H. 1995. Rotifera. The Hague, The Netherlands: SPB Academic. Vol. 2:

The Lecanidae (Monogononta). (Guide to the identification of the microinvertebrates of the continental waters of the world, Vol. 6).

STATSOFT INC. 1991. Statistica. Versão 2.0, Tulsa.

THOMAZ, S.M.; F.A. LANSAC-TÔHA; M.C. RoBerTo; F.A. Esteves \& A.F. LiMA.

1992. Seasonal variation of some limnological factors of a high Paraná River varzea lake - Guaraná Lake - State of Mato Grosso do Sul - Brazil. Rev. Hydrobiol. Trop. 25 (4): 269-276.

VÁsQUEZ, E. 1984a. Estudio de las comunidades de rotíferos del Orinoco Médio,

Bajo Caroni e algunas lagunas de inundación (Venezuela). Mem. Soc. Cienc. Nat. La Salle 44 (121): 95-108.

1984b. El zooplancton de la seccion baja de un rio de aguas negras (Rio

Caroni) y de un embalse hidroelétrico (Macagua I), Venzuela. Mem. Soc. Cienc. Nat. La Salle 44 (121): 109-130.

VÁsQUEZ, E. \&. L. SANCHEZ. 1984. Variación estacional del plancton en dos sectores del rio Orinoco y una laguna del inundación adyacente. Mem. Soc. Cienc. Nat. La Salle 44: 23-31.

WETZEL, R.G. 1990. Land-water interfaces: metabolic and limnological regulators.

Verh. Internat. Verein. Limnol. 24: 6-24.

ZOPPI DE ROA, E.; M.J. PARDO \& W. VÁsQUEZ. 1993. Nuevas adiciones a la fauna de rotíferos de Venezuela. Rev. Hydrobiol. Trop. 26 (3): 165-173.

Recebido em 27.III.1997; aceito em 14.V.1998. 